\title{
Relação entre qualidade de vida e felicidade subjetiva de adolescentes escolares
} Relationship between quality of life and subjective happiness of school adolescents Relación entre calidad de vida y felicidad subjetiva de adolescentes escolares

\author{
Adália Maria Dias Palma Leal (iD \\ Faculdade São Leopoldo Mandic - Campinas (SP) - Brasil \\ Flávia Martão Flório iD \\ Faculdade São Leopoldo Mandic - Campinas (SP) - Brasil \\ Luciane Zanin de Souza iD \\ Faculdade São Leopoldo Mandic - Campinas (SP) - Brasil
}

\section{RESUMO}

Objetivo: Avaliar a qualidade de vida de adolescentes escolares associada aos perfis socioeconômico e demográfico e a relação com felicidade subjetiva. Métodos: Estudo transversal e quantitativo, desenvolvido de dezembro de 2018 a janeiro de 2019, com 339 adolescentes de 14 a 18 anos matriculados no Instituto Federal de Educação, Ciência e Tecnologia do Sertão Pernambucano, em Petrolina, Pernambuco. Avaliaram-se dados relativos aos perfis socioeconômico e demográfico (sexo, idade, renda, moradia, escolaridade da mãe, escola fundamental II, auxílio estudantil, atividade física, curso), assim como verificou-se a qualidade de vida pelo questionário Kidscreen-27 (saúde e atividade física; bem-estar psicológico; autonomia e relação com os pais; amigos e apoio social; ambiente escolar) e a felicidade subjetiva pela Escala de Felicidade Subjetiva. Estimaram odds ratios brutos e ajustados por modelos de regressão logística simples e múltipla, considerando o nível de significância de $5 \%$. Resultados: Amostra composta por 186 (54,9\%) adolescentes do sexo masculino e $153(45,1 \%)$ do sexo feminino, com idade média de 16,4 anos (dp 1,1 ano). Os adolescentes do sexo masculino têm 4,10 vezes (IC95\%: 2,31-7,26) mais chances de ter melhor qualidade de vida em saúde. Adolescentes do curso de Eletrotécnica têm 2,27 vezes (IC95\%: 1,02-5,02) mais chances de ter melhor qualidade de vida em saúde que os de Química $(p<0,05)$. Adolescentes com maior escore de felicidade subjetiva têm 7,10 vezes (IC95\%: 4,18-12,08) mais chances de ter melhor qualidade de vida total em todos os domínios. Conclusão: Fatores como sexo masculino, prática de atividade física, maior renda, menor idade e melhor felicidade subjetiva influenciaram positivamente na qualidade de vida dos adolescentes avaliados.

Descritores: Qualidade de Vida; Saúde do Adolescente; Adulto Jovem; Educação em Saúde.

\section{ABSTRACT}

Objective: To assess the quality of life of school adolescents associated with socioeconomic and demographic profiles and the relationship with subjective happiness. Methods: A cross-sectional and quantitative study, developed from December 2018 to January 2019, with 339 adolescents aged 14 to 18 years enrolled at the Federal Institute of Education, Science, and Technology of the Sertão Pernambucano, in Petrolina, Pernambuco. Data related to socioeconomic and demographic profiles (gender, age, income, housing, mother's education, elementary school II, student aid, physical activity, course) were evaluated, so the quality of life was verified using the Kidscreen-27 questionnaire (health and physical activity; psychological well-being; autonomy and relationship with parents; friends and social support; school environment) and subjective happiness using the Subjective Happiness Scale. They estimated crude odds ratios and adjusted by simple and multiple logistic regression models, considering the significance level of 5\%. Results: This sample consists of 186 (54.9\%) male adolescents and 153 (45.1\%) female adolescents, with a mean age of 16.4 years (SD 1.1 year). Male adolescents are 4.10 times (95\% Cl: 2.31 - 7.26), more likely to have a better quality of life in health. Adolescents in the Electrical Engineering course are 2.27 times (95\% Cl: 1.02-5.02), more likely to have a better quality of life in health than those in Chemistry $(p<0.05)$. Adolescents with a higher subjective happiness score are 7.10 times (95\% Cl: 4.18-12.08), more likely to have a better overall quality of life in all domains. Conclusion: Factors such as male gender, physical activity, higher income, younger age, and better subjective happiness positively influenced the quality of life of evaluated adolescents.

Descriptors: Quality of Life; Adolescent Health; Young Adult; Health Education. 


\section{RESUMEN}

Objetivo: Evaluar la calidad de vida de adolescentes escolares asociada con los perfiles socioeconómicos y demográficos y su relación con la felicidad subjetiva. Métodos: Estudio transversal y cuantitativo desarrollado entre diciembre de 2018 y enero de 2019 con 339 adolescentes entre 14 y 18 años registrados en el Instituto Federal de Educación, Ciencia y Tecnología de la Zona Rural Pernambucana de Petrolina, Pernambuco. Se ha evaluado los datos del perfil socioeconómico y demográfico (sexo, edad, renta, vivienda, escolaridad de la madre, educación infantil II, ayuda de estudiante, actividad física, curso) así como se verificó la calidad de vida por el cuestionario Kidscreen-27 (salud y actividad física; bienestar psicológico; autonomía y relación con los padres; amigos y apoyo social; entorno escolar) y la felicidad subjetiva por la Escala de Felicidad Subjetiva. Se ha estimado odds ratios brutos y ajustados por modelos de regresión logística simple y múltiple considerando el nivel de significación del $5 \%$. Resultados: La muestra ha sido de $186(54,9 \%)$ adolescentes del sexo masculino y $153(45,1 \%)$ del sexo femenino con la edad media de 16,4 años ( $\pm 1,1$ año). Los adolescentes del sexo masculino tienen 4,10 veces (IC95\%: 2,31-7,26) más oportunidades de tener mejor calidad de vida en salud. Adolescentes del curso de Electrotécnica tienen 2,27 veces (IC95\%: 1,02-5,02) más oportunidades de tener mejor calidad de vida en salud que los de Química $(p<0,05)$. Adolescentes con más puntuación de felicidad subjetiva tienen 7,10 veces (IC95\%: 4,18-12,08) más oportunidades de tener mejor calidad de vida total en todos los dominios. Conclusión: Los factores como el sexo masculino, la práctica de actividad física, la mayor renta, la menor edad y la mejor felicidad subjetiva han influenciado de manera positiva en la calidad de vida de los adolescentes evaluados.

Descriptores: Calidad de Vida; Salud del Adolescente; Adulto Joven; Educación en Salud.

\section{INTRODUÇÃO}

A qualidade de vida (QV) é um construto individual, subjetivo, multidimensional e que pode sofrer influência do estado de saúde, nível socioeconômico, estilo de vida, interação social e suporte familiar( ${ }^{(1)}$. A preocupação em avaliar a QV em populações adolescentes destaca-se em razão do seu impacto no desenvolvimento da vida

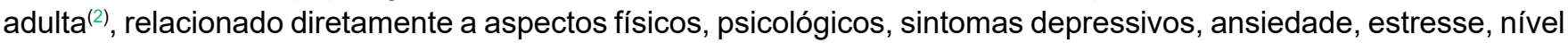
de preocupação e percepção de sua felicidade(3).

Segundo o Estatuto da Criança e do Adolescente (ECA), a adolescência compreende o período de doze e dezoito anos de idade, compreendida entre a infância e a fase adulta, marcada por um complexo processo de crescimento e desenvolvimento biopsicossocial( ${ }^{(4)}$. Estimativa de 2018 indica que a população na faixa etária de 0 a 18 anos era de 68,8 milhões, o que correspondia a $33 \%$ do total da população brasileira ${ }^{(5)}$.

O adolescente está exposto a vários fatores de risco para a saúde dentro do seu contexto social, como o uso de tabaco, o consumo de álcool, a alimentação inadequada e o sedentarismo, que podem alterar a $Q V^{(6)}$.

Com o intuito de proteger e assegurar os direitos dos adolescentes e das crianças foram instituídos programas, como o Programa de Saúde do Adolescente ${ }^{(7)}$, e o $\mathrm{ECA}^{(4)}$, que trouxeram o enfoque de proteção integral para as crianças e os adolescentes que, sem distinção de raça, cor ou classe social, são reconhecidos como sujeitos de direitos. Para nortear ações integradas às outras políticas sanitárias, o Ministério da Saúde (MS) propôs as Diretrizes Nacionais para a Atenção Integral à Saúde de Adolescentes e de Jovens na Promoção, Proteção e Recuperação da Saúde, para que se possa dar um olhar integral às necessidades dessa população( ${ }^{(8)}$.

Como um conjunto de estratégias e formas de produzir saúde, no âmbito individual e coletivo, a promoção da saúde visa atender às necessidades sociais de saúde e garantir a melhoria da QV da população. A saúde é o maior recurso para o desenvolvimento social, econômico e pessoal, assim como uma importante dimensão da QV. É entendida não como um objetivo em si, mas como um recurso fundamental para a vida cotidiana ${ }^{(9)}$. Promover saúde para adolescentes requer fortes ligações intersetoriais que se interconectem com o setor saúde e com a participação e a cooperação de outros setores e da própria comunidade, uma vez que as necessidades de saúde ampliada dessa população perpassam as ações do setor saúde(6)

A adolescência, independente da condição socioeconômica, da faixa etária e do sexo, é uma fase de alta exposição a fatores de risco. Desse modo, iniciativas devem ser direcionadas para que haja eficiente ação educativa, preventiva e assistencial, garantindo a promoção da saúde ${ }^{(10)}$. A promoção de saúde com adolescentes deve estar associada a uma educação construtiva, libertadora, dialógica e promotora de sua autonomia no autocuidado. Novos paradigmas surgiram na área da saúde, de modo que o modelo hegemônico centrado na doença dá lugar a uma lógica que prioriza a QV das pessoas ${ }^{(11)}$.

Nos últimos anos, tem sido observado um aumento no número de publicações sobre QV de adolescentes no Brasil e em outros países. Entre os países que mais relizaram estudos, destacam-se a Alemanha, a Espanha, a Holanda e Portugal. Na América do Sul, destacam-se o Chile e o Brasil ${ }^{(12)}$. 
Da mesma forma que a QV, a felicidade, definida como satisfação, estado de espírito, afeto positivo e bem-estar ${ }^{(13)}$, baseia-se em um constructo multidimensional. É também afetada por fatores externos, como renda, trabalho, comunidade e governança, valores e religião, e por fatores pessoais, como saúde mental e física, experiência familiar, educação, idade e sexo(14).

Entender como o adolescente da região Nordeste do Brasil, dentro do seu contexto social, econômico e cultural, percebe sua $Q V$ e quais fatores podem estar relacionados a uma visão positiva ou negativa de sua vida poderá contribuir para o planejamento de ações interventivas que considerem como prioridade a educação em saúde. Dessa forma, o presente objetivou avaliar a qualidade de vida de adolescentes escolares associada aos perfis socioeconômico e demográfico e a relação com felicidade subjetiva.

\section{MÉTODOS}

Estudo epidemiológico, observacional, descritivo e transversal desenvolvido com adolescentes do Instituto Federal de Educação, Ciência e Tecnologia do Sertão Pernambucano (IF Sertão-PE), localizado no município de Petrolina, Pernambuco.

O IF Sertão-PE é uma instituição de educação superior, básica e profissional, pluricurricular e multicampi. Promove a inclusão social e reduz vulnerabilidades, sendo uma ilha de excelência do ensino público do Brasil. $O$ acesso aos cursos técnicos realiza-se por meio de um processo seletivo anual. Na modalidade médio integrado são oferecidos os cursos de Edificações, Eletrotécnica, Informática e Química ${ }^{(15)}$.

O presente estudo desenvolveu-se de dezembro de 2018 a janeiro de 2019. Para participar da pesquisa convidaram-se todos os 493 adolescentes matriculados no IF Sertão-PE no ano de 2018 nos cursos médio integrado - Informática (65 alunos), Edificações (103 alunos), Eletrotécnica (71 alunos) e Química (100 alunos).

Para a inclusão dos adolescentes no estudo, considerou-se todos com idade de 14 a 18 anos que concordaram em participar e assinaram o Termo de Consentimento Livre e Esclarecido e o Termo de Assentimento. Considerou-se como critério de exclusão os adolescentes que não completaram o preenchimento do questionário ou que não estavam presentes no dia da coleta de dados do estudo.

Coletaram-se os dados a partir dos questionários impressos, aplicados durante o período regular de aula e na presença somente da pesquisadora responsável para esclarecer dúvidas sobre o preenchimento, garantindo a qualidade dos dados. Os adolescentes responderam aos questionários de forma única, direta e objetiva.

Como variáveis independentes, considerou-se o perfil sociodemográfico: curso (Informática, Edificações, Eletrotécnica e Química); sexo (feminino e masculino); idade (14 a 18 anos); renda baseada no número de salários mínimos (SM), no valor de 954,00 reais (classe A - acima de $20 \mathrm{SM}$, classe B - de 10 a $20 \mathrm{SM}$, classe C - de 4 a $10 \mathrm{SM}$, classe D - de 2 a 4 SM e classe E - até $2 \mathrm{SM}$ ); tipo de moradia (própria, alugada, herança, cedida ou financiada); escolaridade da mãe (analfabeta, $1^{\circ}$ grau incompleto, $1^{\circ}$ grau completo, $2^{\circ}$ grau incompleto, $2^{\circ}$ grau completo ou nível superior); recebimento ou não do auxilio financeiro da política estudantil; número de auxílios recebidos; pratica de atividade física; tipo de escola em que cursou o fundamental II (pública, particular, particular e pública); felicidade subjetiva.

Em relação à avaliação da felicidade subjetiva, empregou-se a escala Escala de Felicidade Subjetiva (SHS), a qual avalia de forma global a felicidade subjetiva variando de 1 a 7 pontos. No presente estudo, utilizou-se o escore global da escala considerando que pontuações mais altas correspondem a uma maior felicidade subjetiva ${ }^{(16)}$.

Para a coleta de dados referente a QV, utilizou-se o questionário autoadministrado Kidscreen-27, criado de forma específica para crianças e adolescentes, com 27 itens e múltipla escolha distribuídos em cinco domínios da QV: saúde e atividade física (percepção sobre a condição física), bemestar psicológico (percepção sobre a condição afetiva e cognitiva), autonomia e relação com os pais (percepção sobre o relacionamento com os pais), amigos e apoio social (percepção sobre a relação entre pares) e ambiente escolar (percepção sobre a escola e capacidade escolar). A QV total varia de 0 a 100, sendo classificada em: valores entre 45 e 55 - QV normal, valores abaixo de 45 - QV negativa/ruim e valores acima de 55 - QV positiva/boa ${ }^{(17)}$.

Utilizou-se estatística descritiva a partir da distribuição da frequências simples e relativas para a realização da análise dos dados. Em seguida, analisaram-se as associações entre cada variável de desfecho e as variáveis independentes. Estimou-se, também, os odds ratio (OR) brutos com os respectivos intervalos de $95 \%$ de confiança, para isso utilizou-se a análise de regressão logística simples. Estudaram-se as variáveis com $p<0,20$ nas análises brutas em modelos de regressão logística múltipla, mantendo nos modelos finais aquelas com $p \leq 0,05$.

Estimaram-se pelos modelos de regressão múltipla os OR ajustados com os respectivos intervalos de $95 \%$ de confiança. O OR bruto representa o grau da associação para cada variável individualmente, sem considerar as 
demais. O OR ajustado representa o grau da associação para as variáveis que permaneceram no modelo final, ajustadas entre si. Para fins das análise, dicotomizou-se as variáveis idade, renda, escore de felicidade subjetiva e escore do Kidscreen-27 pela mediana a fim de garantir o balanceamento das categorias.

Conduziu-se a pesquisa de acordo com os preceitos determinados pela Resolução n. ${ }^{\circ} 466 / 12$, para estudo com seres humanos, do Conselho Nacional de Saúde do Ministério da Saúde e, quando submetido à apreciação, o projeto do estudo recebeu aprovação pelo Comitê de Ética e Pesquisa da Faculdade São Leopoldo Mandic, Campinas (São Paulo), sob o Parecer n. ${ }^{\circ}$ 3.024.931.

\section{RESULTADOS}

Dos 493 adolescentes, $339(68,8 \%)$ responderam aos questionários, correspondendo a uma perda amostral de $154(31,2 \%)$. Observou-se $186(54,9 \%)$ adolescentes do sexo masculino e $153(45,10)$ do sexo feminino; média de idade de 16,4 anos; $172(50,7 \%)$ da classe E; $237(69,9 \%)$ residentes em moradia própria; $277(81,7 \%)$ das mães com escolaridade maior que o $1^{\circ}$ grau e $151(52,2 \%)$ adolescentes recebem auxílio financeiro.

Tratando-se da QV total dos adolescentes, observou-se o valor médio de 65,5. Em relação aos domínios avaliados, percebeu-se a maior média para o domínio relativo a amigos e apoio social $(69,5)$. Tratando-se da felicidade subjetiva, verificou-se média de 4,68, como apresentado na Tabela I.

Tabela I - Análise descritiva da qualidade de vida relacionada à saúde (Kidscreen-27) de adolescentes do Instituto Federal de Educação, Ciência e Tecnologia do Sertão Pernambucano (IF SertãoPE). Petrolina, Pernambuco, Brasil, 2019.

\begin{tabular}{lccccc}
\hline Variáveis & Média & Desvio padrão & Mediana & Mínimo & Máximo \\
\hline Saúde e atividade física & 59,9 & 16 & 60 & 24 & 100 \\
Bem-estar psicológico & 66,7 & 15 & 68,6 & 25,7 & 97,1 \\
Autonomia e relação com os pais & 64,9 & 14,7 & 65,7 & 25,7 & 100 \\
Amigos e apoio social & 69,5 & 15,4 & 70 & 25 & 100 \\
Ambiente escolar & 67,5 & 13 & 70 & 20 & 100 \\
${ }^{*}$ Qualidade de vida total & 65,5 & 11,3 & 65,8 & 37,3 & 96,1 \\
$* *$ Felicidade subjetiva & 4,68 & 1,23 & 4,75 & 1 & 7 \\
\hline
\end{tabular}

* valor que varia de 0 a 100; ** valor que varia de 0 a 7

Nas Tabelas II e III são apresentados os resultados entre os escores dos domínios do instrumento Kidscreen-27 e as variáveis demográficas e socioeconômicas e de felicidade subjetiva.

De acordo com os resultados apresentados em relação ao domínio saúde e atividade física, observou-se que adolescentes que são do sexo masculino, que praticam atividade física e que possuem maior escore de felicidade subjetiva têm 6,37 (IC95\%: 3,49-11,63), 9,97 (IC95\%: 4,62-21,50) e 3,00 (IC95\%: 1,68-5,34) vezes mais chances, respectivamente, de ter melhor QV no domínio saúde e atividade física $(p<0,05)$.

Adolescentes do sexo masculino e com maior escore de felicidade subjetiva têm 4,65 (IC95\%: 2,59-8,33) e 15,74 (IC95\%: 8,85-27,99) vezes mais chances, respectivamente, de ter maior QV no domínio bem-estar psicológico $(p<0,05)$, como consta na Tabela II.

Pode-se observar, também, que os alunos dos cursos de Edificações e de Eletrotécnica têm 2,24 (IC95\%: 1,22-4,10) e 3,27 (IC95\%: 1,58-6,78) vezes mais chances, respectivamente, de ter maior QV no domínio autonomia e relação com os pais do que os alunos de Química $(p<0,05)$. Os adolescentes do sexo masculino, de idade até 16 anos e com maior escore de felicidade subjetiva têm 2,33 (IC95\%: 1,40-3,89), 1,71 (IC95\%: 1,07-2,72) e 2,03 (IC95\%: 1,27-3,25) vezes mais chances, respectivamente, de ter melhor $Q V$ nesse domínio $(p<0,05)$, como apresenta a Tabela III.

Em relação aos resultados observados no domínio amigos e apoio social, alunos do curso de Eletrotécnica têm 2,23 (IC95\%: 1,15-4,31) vezes mais chances de ter melhor QV do que os alunos do curso de Química. Estudantes de classe econômica D e com maior escore de felicidade subjetiva têm 1,62 (IC95\%: 1,02-2,56) e 3,63 (IC95\%: 
$2,29-5,77)$ vezes mais chances, respectivamente, de ter melhor $Q V$ nesse domínio $(p<0,05)$. Estudantes do sexo masculino e com maior escore de felicidade subjetiva têm 2,03 (IC95\%: 1,26-3,26) e 2,92 (IC95\%: 1,83-4,65) vezes mais chances, respectivamente, de ter melhor QV no domínio ambiente escolar, como dispõe a Tabela III.

Tabela II - Análises (bruta e ajustada) das associações entre escores dos domínios Saúde e atividade física e Bemestar psicológico avaliados pelo Kidscreen-27 e as variáveis demográficas, socioeconômicas e de felicidade de adolescentes do IF SertãoPE. Petrolina, Pernambuco, Brasil, 2019.

\begin{tabular}{|c|c|c|c|c|c|}
\hline \multirow[b]{3}{*}{ Variável } & \multirow[b]{3}{*}{ Categoria } & \multicolumn{4}{|c|}{ Domínios } \\
\hline & & \multicolumn{2}{|c|}{ Saúde e atividade física } & \multicolumn{2}{|c|}{ Bem-estar psicológico } \\
\hline & & $\begin{array}{l}\text { OR bruto } \\
\text { (IC95\%) }\end{array}$ & $\begin{array}{l}\text { OR ajustado } \\
\text { (IC95\%) }\end{array}$ & $\begin{array}{l}\text { OR bruto } \\
\text { (IC95\%) }\end{array}$ & $\begin{array}{l}\text { OR ajustado } \\
\text { (IC95\%) }\end{array}$ \\
\hline \multirow{4}{*}{ Curso } & Edificações & $1,53(0,86-2,73)$ & - & $1,66(0,93-2,95)$ & - \\
\hline & Eletrotécnica & **3,04 $(1,61-5,73)$ & - & **3,63 $(1,91-6,88)$ & - \\
\hline & Informática & **2,44 (1,28-4,65) & - & *1,91 (1,00-3,64) & - \\
\hline & Química & Ref & - & Ref & - \\
\hline \multirow{2}{*}{ Sexo } & Feminino & Ref & Ref & Ref & Ref \\
\hline & Masculino & **7,12 $(4,32-11,71)$ & ${ }^{* *} 6,37(3,49-11,63)$ & $* * 3,78(2,38-6,00)$ & **4,65 $(2,59-8,33)$ \\
\hline \multirow{2}{*}{ Idade } & $\leq$ Mediana (16 anos) & $1,33(0,87-2,06)$ & - & $1,30(0,85-2,00)$ & - \\
\hline & $>$ Mediana & Ref & - & Ref & - \\
\hline \multirow{2}{*}{ Renda } & $\leq$ Mediana (Classe D) & $1,10(0,72-1,70)$ & - & $0,98(0,64-1,51)$ & - \\
\hline & > Mediana (Classe E) & Ref & - & Ref & - \\
\hline \multirow{2}{*}{ Moradia } & Própria & $1,43(0,88-2,31)$ & - & $1,60(0,98-2,58)$ & - \\
\hline & Não própria & Ref & - & Ref & - \\
\hline \multirow{2}{*}{ Escolaridade da mãe } & Até o $1^{\circ}$ grau completo & Ref & - & Ref & - \\
\hline & Mais anos de estudo & $1,06(0,60-1,90)$ & - & $0,75(0,42-1,34)$ & - \\
\hline \multirow{2}{*}{$\begin{array}{l}\text { Auxílio financeiro da } \\
\text { política estudantil no IF }\end{array}$} & Sim & $0,9841(0,64-1,52)$ & - & $1,35(0,87-2,08)$ & - \\
\hline & Não & Ref & - & Ref & - \\
\hline \multirow{3}{*}{$\begin{array}{l}\text { Quantos auxílios } \\
\text { financeiros da política } \\
\text { estudantil no IF }\end{array}$} & 1 & $1,05(0,65-1,69)$ & - & $0,72(0,45-1,16)$ & - \\
\hline & 2 ou mais & $0,87(0,44-1,75)$ & - & $0,75(0,37-1,50)$ & - \\
\hline & Não recebe & Ref & - & Ref & - \\
\hline \multirow{4}{*}{ Tipo de escola } & Pública & Ref & - & Ref & - \\
\hline & Particular sem bolsa & $1,30(0,77-2,20)$ & - & $1,50(0,36-1,95)$ & - \\
\hline & Particular com bolsa & $0,94(0,41-2,15)$ & - & $0,84(0,36-1,95)$ & - \\
\hline & Particular e pública & $0,98(0,54-1,77)$ & - & $1,17(0,65-2,13)$ & - \\
\hline \multirow{3}{*}{ Atividade física } & Esporadicamente & *2,40 (1,10-5,23) & $1,32(0,56-3,11)$ & $1,71(0,91-3,21)$ & - \\
\hline & Não & Ref & Ref & Ref & - \\
\hline & Sim & **14,08 $(6,98-28,39)$ & ${ }^{* *} 9,97(4,62-21,50)$ & **2,33 $(1,34-4,07)$ & - \\
\hline \multirow{2}{*}{$\begin{array}{l}\text { Escala de felicidade } \\
\text { subjetiva }\end{array}$} & $\leq$ Mediana $(4,75)$ & Ref & Ref & Ref & Ref \\
\hline & $>$ Mediana & **2,88 $(1,84-4,49)$ & **3,00 $(1,68-5,34)$ & **13,89 $(8,22-23,48)$ & **15,74 $(8,85-27,99)$ \\
\hline
\end{tabular}

${ }^{*} \mathrm{p} \leq 0,05 ;{ }^{* *} \mathrm{p} \leq 0,01$; OR: Odds ratio; IC: Intervalo de confiança; Ref: Categoria de referência de cada variável independente; IF SertãoPE: Instituto Federal de Educação, Ciência e Tecnologia do Sertão Pernambucano 
Tabela III - Análises (bruta e ajustada) das associações entre os escores dos domínios Autonomia e relação com os pais, Amigos e apoio social e Ambiente escolar avaliados pelo Kidscreen-27 e as variáveis demográficas, socioeconômicas e de felicidade de adolescentes do IF SertãoPE. Petrolina, Pernambuco, Brasil, 2019.

\begin{tabular}{|c|c|c|c|c|c|c|c|}
\hline \multirow[b]{3}{*}{ Variável } & \multirow[b]{3}{*}{ Categoria } & \multicolumn{6}{|c|}{ Domínios } \\
\hline & & \multicolumn{2}{|c|}{$\begin{array}{c}\text { Autonomia e relação com os } \\
\text { pais }\end{array}$} & \multicolumn{2}{|c|}{ Amigos e apoio social } & \multicolumn{2}{|c|}{ Ambiente escolar } \\
\hline & & $\begin{array}{l}\text { OR bruto } \\
\text { (IC95\%) }\end{array}$ & $\begin{array}{l}\text { OR ajustado } \\
\text { (IC95\%) }\end{array}$ & $\begin{array}{l}\text { OR bruto } \\
\text { (IC95\%) }\end{array}$ & $\begin{array}{l}\text { OR ajustado } \\
\text { (IC95\%) }\end{array}$ & $\begin{array}{l}\text { OR bruto } \\
\text { (IC95\%) }\end{array}$ & $\begin{array}{l}\text { OR ajustado } \\
\text { (IC95\%) }\end{array}$ \\
\hline \multirow{4}{*}{ Curso } & Edificações & $" 2,40(1,34-4,29)$ & "2,24 (1,22-4,10) & $1,27(0,72-2,23)$ & $1,19(0,65-2,16)$ & $1,36(0,76-2,45)$ & - \\
\hline & Eletrotécnica & $" 5,45(2,81-10,58)$ & "3,27 (1,58-6,78) & "2,47 (1,32-4,60) & $2,23(1,15-4,31)$ & $1,71(0,90-3,22)$ & - \\
\hline & Informática & $" 2,68(1,40-5,15)$ & $2,02(0,99-4,10)$ & $1,29(0,68-2,44)$ & $1,27(0,65-2,48)$ & $1,37(0,70-2,64)$ & - \\
\hline & Química & Ref & Ref & Ref & Ref & Ref & - \\
\hline \multirow{2}{*}{ Sexo } & Feminino & Ref & Ref & Ref & - & Ref & Ref \\
\hline & Masculino & $" 3,19(2,04-4,99)$ & "2,33 (1,40-3,89) & $1,28(0,83-1,98)$ & - & "2,23 (1,40-3,54) & "2,03 (1,26-3,26) \\
\hline \multirow{2}{*}{ Idade } & $\leq$ Mediana (16 anos) & $\cdot 1,64(1,06-2,51)$ & $1,71(1,07-2,72)$ & $1,02(0,67-1,57)$ & - & $1,32(0,84-2,06)$ & - \\
\hline & > Mediana & Ref & Ref & Ref & - & Ref & - \\
\hline \multirow{2}{*}{ Renda } & $\leq$ Mediana $($ Classe D) & $1,51(0,98-2,32)$ & - & $1,51(0,98-2,32)$ & *1,62 (1,02-2,56) & $0,81(0,52-1,26)$ & - \\
\hline & > Mediana (Classe E) & Ref & - & Ref & Ref & Ref & - \\
\hline \multirow{2}{*}{ Moradia } & Própria & $1,29(0,81-2,07)$ & - & $1,41(0,88-2,28)$ & - & $1,34(0,82-2,21)$ & - \\
\hline & Não própria & Ref & - & Ref & - & Ref & - \\
\hline \multirow{2}{*}{ Escolaridade da mãe } & Até o $1^{\circ}$ grau completo & Ref & - & Ref & - & Ref & - \\
\hline & Mais anos de estudo & $1,08(0,61-1,92)$ & - & $0,92(0,52-1,64)$ & - & $0,70(0,39-1,27)$ & - \\
\hline \multirow{2}{*}{$\begin{array}{l}\text { Auxilio financeiro da } \\
\text { política estudantil no IF }\end{array}$} & Sim & $1,07(0,70-1,64)$ & - & $0,80(0,52-1,24)$ & - & $0,75(0,48-1,18)$ & - \\
\hline & Não & Ref & - & Ref & - & Ref & - \\
\hline \multirow{3}{*}{$\begin{array}{l}\text { Quantos auxílios } \\
\text { financeiros da política } \\
\text { estudantil no IF }\end{array}$} & 1 & $0,98(0,61-1,57)$ & - & $0,81(0,50-1,30)$ & - & $1,50(0,93-2,44)$ & - \\
\hline & 2 ou mais & $0,86(0,44-1,71)$ & - & $0,75(0,37-1,50)$ & - & $0,86(0,41-1,81)$ & - \\
\hline & Não recebe & Ref & - & Ref & - & Ref & - \\
\hline \multirow{4}{*}{ Tipo de escola } & Pública & Ref & - & Ref & - & Ref & - \\
\hline & Particular sem bolsa & $1,65(0,98-2,80)$ & - & $1,00(0,59-1,69)$ & - & $1,13(0,66-1,93)$ & - \\
\hline & Particular com bolsa & $1,80(0,79-4,12)$ & - & $0,68(0,29-1,57)$ & - & $0,45(0,17-1,18)$ & - \\
\hline & Particular e pública & $0,83(0,45-4,50)$ & & $0,77(0,42-1,40)$ & - & $0,58(0,30-1,10)$ & - \\
\hline \multirow{3}{*}{ Atividade física } & Esporadicamente & $1,46(0,79-2,69)$ & - & $1,03(0,55-1,91)$ & - & $1,41(0,74-2,69)$ & - \\
\hline & Não & Ref & - & Ref & - & Ref & - \\
\hline & Sim & $1,59(0,93-2,72)$ & - & $1,51(0,88-2,58)$ & - & $1,65(0,94-2,92)$ & - \\
\hline \multirow{2}{*}{$\begin{array}{l}\text { Escala de felicidade } \\
\text { subjetiva }\end{array}$} & $\leq$ Mediana $(4,75)$ & Ref & Ref & Ref & Ref & Ref & Ref \\
\hline & $>$ Mediana & "2,27 (1,46-3,52) & "2,03 (1,27-3,25) & "3,67 (2,33-5,77) & "3,63 (2,29-5,77) & "3,10 (1,96-4,92) & "2,92 (1,83-4,65) \\
\hline
\end{tabular}

${ }^{*} \mathrm{p} \leq 0,05 ;{ }^{*} \mathrm{p} \leq 0,01$; OR: Odds ratio; IC: Intervalo de confiança; Ref: Categoria de referência de cada variável independente; IF SertãoPE: Instituto Federal de Educação, Ciência e Tecnologia do Sertão Pernambucano

As Tabelas IV e $\mathrm{V}$ apresentam, respectivamente, o resultado das análises bruta e ajustada das associações entre o escore do Kidscreen-27 e as variáveis demográficas, socioeconômicas e de felicidade. De acordo com a Tabela IV, as variáveis curso, sexo, atividade física e felicidade mostraram-se associadas à variável dependente na análise bruta, no entanto, após ajuste entre as variáveis, ou seja, entudando-se as variáveis em conjunto, pôde-se observar que estudantes do sexo masculino e com maior escore de felicidade subjetiva têm 4,10 (IC95\%: 2,31-7,26) e 7,10 (IC95\%: 4,18-12,08) vezes mais chances, respectivamente, de ter melhor QV em saúde $(p<0,05)$. Além, disso, os alunos de Eletrotécnica têm 2,27 (IC95\%: 1,02-5,02) vezes mais chances de ter melhor QV que os alunos do curso de Química $(p<0,05)$, como consta na Tabela V. 
Tabela IV - Análise bruta das associações entre o escore total da qualidade de vida em saúde avaliada pelo Kidscreen-27 e as variáveis demográficas, socioeconômicas e de felicidade de adolescentes do IF SertãoPE. Petrolina, Pernambuco, Brasil, 2019.

\begin{tabular}{|c|c|c|c|c|c|c|}
\hline & & & \multicolumn{2}{|c|}{$\begin{array}{c}\text { Questionário Kidscreen-27 - } \\
\text { Total }\end{array}$} & \multirow[t]{2}{*}{ OR bruto (IC95\%) } & \multirow[t]{2}{*}{ p-valor } \\
\hline & & & $\leq$ Mediana $(65,8)$ & $>$ Mediana* & & \\
\hline Variável & Categoria & n (\%) & n (\%) & n (\%) & & \\
\hline \multirow{4}{*}{ Curso } & Edificações & $103(30,4 \%)$ & $54(52,4 \%)$ & $49(47,6 \%)$ & $1,76(1,00-3,01)$ & 0,0501 \\
\hline & Eletrotécnica & $71(20,9 \%)$ & $21(29,6 \%)$ & $50(70,4 \%)$ & $4,62(2,40-8,91)$ & $<0,0001$ \\
\hline & Informática & $65(19,2 \%)$ & $30(46,2 \%)$ & $35(53,8 \%)$ & $2,26(1,20-4,29)$ & 0,0122 \\
\hline & Química & $100(29,5 \%)$ & $66(66,0 \%)$ & $34(34,0 \%)$ & Ref & \\
\hline \multirow{2}{*}{ Sexo } & Feminino & $153(45,1 \%)$ & $108(70,6 \%)$ & $45(29,4 \%)$ & Ref & \\
\hline & Masculino & $186(54,9 \%)$ & $63(33,9 \%)$ & $123(66,1 \%)$ & $4,69(2,95-7,44)$ & $<0,0001$ \\
\hline \multirow{2}{*}{ Idade } & $\leq$ Mediana (16 anos) & $174(51,3 \%)$ & $85(48,9 \%)$ & $89(51,1 \%)$ & $1,14(0,74-1,74)$ & 0,5473 \\
\hline & $>$ Mediana & $165(48,7 \%)$ & $86(52,1 \%)$ & $79(47,9 \%)$ & Ref & \\
\hline \multirow{2}{*}{ Renda } & $\leq$ Mediana (Classe D) & $167(49,3 \%)$ & $78(46,7 \%)$ & $89(53,3 \%)$ & $1,34(0,88-2,06)$ & 0,1756 \\
\hline & > Mediana (Classe E) & $172(50,7 \%)$ & $93(54,1 \%)$ & $79(45,9 \%)$ & Ref & \\
\hline \multirow{3}{*}{ Moradia } & Própria & $237(69,9 \%)$ & $114(48,1 \%)$ & $123(51,9 \%)$ & $1,40(0,88-2,25)$ & 0,1579 \\
\hline & Não própria & $99(29,2 \%)$ & $56(56,6 \%)$ & $43(43,4 \%)$ & Ref & \\
\hline & Não respondeu & $3(0,9 \%)$ & $1(33,3 \%)$ & $2(66,7 \%)$ & - & \\
\hline \multirow{3}{*}{ Escolaridade da mãe } & Até o $1^{\circ} \mathrm{grau}$ completo & $56(16,5 \%)$ & $26(46,4 \%)$ & $30(53,6 \%)$ & Ref & \\
\hline & Outros & $277(81,7 \%)$ & $141(50,9 \%)$ & $136(49,1 \%)$ & $0,84(0,47-1,49)$ & 0,5414 \\
\hline & Não respondeu & $6(1,8 \%)$ & $4(66,7 \%)$ & $2(33,3 \%)$ & - & \\
\hline \multirow{3}{*}{$\begin{array}{l}\text { Auxílio financeiro da } \\
\text { política estudantil no IF }\end{array}$} & Sim & $187(55,2 \%)$ & $91(48,7 \%)$ & $96(51,3 \%)$ & $1,19(0,77-1,83)$ & 0,4301 \\
\hline & Não & $151(44,5 \%)$ & $80(53,0 \%)$ & $71(47,0 \%)$ & Ref & \\
\hline & Não respondeu & $1(0,3 \%)$ & $0(0,0 \%)$ & $1(100,0 \%)$ & - & \\
\hline \multirow{4}{*}{$\begin{array}{l}\text { Quantos auxílios } \\
\text { financeiros da política } \\
\text { estudantil no IF }\end{array}$} & 1 & $110(32,4 \%)$ & $55(50,0 \%)$ & $55(50,0 \%)$ & $0,948(0,59-1,52)$ & 0,8239 \\
\hline & 2 ou mais & $40(11,8 \%)$ & $25(62,5 \%)$ & $15(37,5 \%)$ & $0,57(0,28-1,15)$ & 0,1148 \\
\hline & Não recebe & $187(55,2 \%)$ & $91(48,7 \%)$ & $96(51,3 \%)$ & Ref & \\
\hline & Não respondeu & $2(0,6 \%)$ & $0(0,0 \%)$ & $2(100,0 \%)$ & - & \\
\hline \multirow{4}{*}{ Tipo de escola } & Pública & $168(49,6 \%)$ & $90(53,6 \%)$ & $78(46,4 \%)$ & Ref & \\
\hline & Particular sem bolsa & $84(24,8 \%)$ & $35(41,7 \%)$ & $49(58,3 \%)$ & $1,62(0,95-2,74)$ & 0,0757 \\
\hline & Particular com bolsa & $27(8,0 \%)$ & $14(51,9 \%)$ & $13(48,1 \%)$ & $1,07(0,48-2,42)$ & 0,8680 \\
\hline & Particular e pública & $60(17,7 \%)$ & $32(53,3 \%)$ & $28(46,7 \%)$ & $1,01(0,56-1,82)$ & 0,9747 \\
\hline \multirow{4}{*}{ Atividade física } & Esporadicamente & $82(24,2 \%)$ & $45(54,9 \%)$ & $37(45,1 \%)$ & $1,54(0,82-2,86)$ & 0,1764 \\
\hline & Não & $86(25,4 \%)$ & $56(65,1 \%)$ & $30(34,9 \%)$ & Ref & \\
\hline & Sim & $151(44,5 \%)$ & $59(39,1 \%)$ & $92(60,9 \%)$ & $2,91(1,68-5,05)$ & 0,0001 \\
\hline & Não respondeu & $20(5,9 \%)$ & $11(55,0 \%)$ & $9(45,0 \%)$ & - & \\
\hline \multirow{2}{*}{$\begin{array}{l}\text { Escala de felicidade } \\
\text { subjetiva }\end{array}$} & $\leq$ Mediana $(4,75)$ & $193(56,9 \%)$ & $134(69,4 \%)$ & $59(30,6 \%)$ & Ref & \\
\hline & $>$ Mediana & $146(43,1 \%)$ & $37(25,3 \%)$ & $109(74,7 \%)$ & $6,69(4,13-10,84)$ & $<0,0001$ \\
\hline
\end{tabular}

"Categoria de referência para a variável de desfecho; OR: Odds ratio; IC: Intervalo de confiança; Ref: Categoria de referência de cada variável independente; IF SertãoPE: Instituto Federal de Educação, Ciência e Tecnologia do Sertão Pernambucano 
Tabela $V$ - Análise ajustada das associações entre o escore total da qualidade de vida em saúde avaliada pelo Kidscreen-27 e as variáveis demográficas, socioeconômicas e de felicidade de adolescentes do IF SertãoPE. Petrolina, Pernambuco, Brasil, 2019.

\begin{tabular}{|c|c|c|c|c|c|c|}
\hline \multirow[b]{3}{*}{ Variável } & \multirow[b]{3}{*}{ Categoria } & \multirow[b]{3}{*}{ n (\%) } & \multicolumn{2}{|c|}{ Questionário Kidscreen-27 - Total } & \multirow[t]{3}{*}{ OR ajustado (IC95\%) } & \multirow[t]{3}{*}{ p-valor } \\
\hline & & & $\leq$ Mediana $(65,8)$ & $>$ Mediana* & & \\
\hline & & & n (\%) & $\mathrm{n}(\%)$ & & \\
\hline \multirow{4}{*}{ Curso } & Edificações & $103(30,4 \%)$ & $54(52,4 \%)$ & $49(47,6 \%)$ & $1,45(0,74-2,83)$ & 0,2742 \\
\hline & Eletrotécnica & $71(20,9 \%)$ & $21(29,6 \%)$ & $50(70,4 \%)$ & $2,27(1,02-5,02)$ & 0,0434 \\
\hline & Informática & $65(19,2 \%)$ & $30(46,2 \%)$ & $35(53,8 \%)$ & $1,48(0,68-3,25)$ & 0,3197 \\
\hline & Química & $100(29,5 \%)$ & $66(66,0 \%)$ & $34(34,0 \%)$ & Ref & \\
\hline \multirow{2}{*}{ Sexo } & Feminino & $153(45,1 \%)$ & $108(70,6 \%)$ & $45(29,4 \%)$ & Ref & \\
\hline & Masculino & $186(54,9 \%)$ & $63(33,9 \%)$ & $123(66,1 \%)$ & $4,10(2,31-7,26)$ & $<0,0001$ \\
\hline \multirow[t]{2}{*}{ Escala de felicidade subjetiva } & $\leq$ Mediana $(4,75)$ & $193(56,9 \%)$ & $134(69,4 \%)$ & $59(30,6 \%)$ & Ref & \\
\hline & $>$ Mediana & $146(43,1 \%)$ & $37(25,3 \%)$ & $109(74,7 \%)$ & $7,10(4,18-12,08)$ & $<0,0001$ \\
\hline
\end{tabular}

"Categoria de referência para a variável de desfecho; OR: Odds ratio; IC: Intervalo de confiança; Ref: Categoria de referência de cada variável independente; IF SertãoPE: Instituto Federal de Educação, Ciência e Tecnologia do Sertão Pernambucano

\section{DISCUSSÃO}

A percepção da QV e da felicidade subjetiva demonstrou-se satisfatória no grupo de adolescentes avaliado, visto que considerou-se tanto os escores da QV total quanto de seus domínios de forma positiva/boa ${ }^{(17)}$. Observou-se que sexo, tipo de curso e felicidade subjetiva apresentaram a maior interferência na QV dos adolescentes avaliados.

A literatura aponta estudos em adolescentes que mostraram resultados semelhantes ${ }^{(18-20)}$. Estudos realizados no Chile e na Colômbia apresentaram menor percepção da $\mathrm{QV}^{(21,22)}$, o que mostra que a dinâmica envolvida nos contextos social, econômico e cutural dos adolescentes (família, escola, pares, comunidade, renda) pode influenciar as diversas facetas da $\mathrm{QV}^{(23)}$.

Em todos os domínios observados, os adolescentes classificaram a QV como positiva, não sendo possível apontar nenhum domínio que tenha contribuído de forma negativa na QV geral. No domínio amigos e apoio social obteve-se a maior pontuação $(69,5)$. Outros estudos da literatura mostraram alta pontuação nesse domínio ${ }^{(24,25)}$, evidenciando que os adolescentes do IF Sertão-PE também apresentam boas relações de amizade, fundamentais para o desenvolvimento social, emocional e cognitivo nessa fase da vida(25).

O segundo domínio com a maior pontuação relaciona-se ao ambiente escolar $(67,5)$, demonstrando que os adolescentes percebem a escola como um ambiente favorável para a sua QV. Assim, a escola constitui local privilegiado para o monitoramento de fatores de risco e proteção dos adolescentes, pois, além do papel de formadores de valores, hábitos e estilo de vida, é o local que reúne as melhores condições para a implementação de ações de promoção de saúde ${ }^{(26)}$.

Analisando quais fatores poderiam interferir em cada um dos domínios, verificou-se que ser do sexo masculino aumentou a chance de ter maior QV total em 4 dos 5 domínios avaliados, o que corrobora com a evidência de que o sexo masculino apresenta uma percepção mais positiva da vida, já que as meninas tendem a ser mais exigentes em relação à percepção de si mesmas ${ }^{(27,28)}$.

A prática de atividade física também aumentou em 9,97 vezes a chance de ter melhor $Q V$ relacionada ao domínio saúde e atividade física, pois proporciona ao indivíduo inúmeros benefícios, como melhora no bem-estar, autoestima, autoconfiança e interação social, que, por sua vez, ajudam na melhora das capacidades físicas, mentais, afetivas, sociais, contribuindo na prevenção e tratamento de doenças ${ }^{(29)}$. Em contraponto, um estudo de abrangência nacional concluiu que mais da metade dos adolescentes brasileiros não atingem a recomendação mínima de 300 minutos/ semana de atividade física, sendo esse percentual ainda maior entre as meninas, ultrapassando $70 \%{ }^{(30)}$, o que pode ajudar a compreender os resultados verificados no sexo masculino do presente estudo.

Com o intuito de promover hábitos saudáveis, visando a prevenção de doenças crônicas e a consequente melhoria da QV dos brasileiros, o MS implementou estratégias específicas voltadas ao adolescente, como as Escolas Promotoras de Saúde, o Programa Academia da Saúde, a Pesquisa Nacional de Saúde do Escolar e o Programa Saúde na Escola. Tratam-se de iniciativas voltadas para incentivar a prática da atividade física, educação em saúde 
e de cuidados à saúde, de forma a incentivar as praticas saúdáveis que interferem positivamente na promoção de saúde desse grupo ${ }^{(31)}$.

A idade também interferiu na $Q V$, revelando que ter idade até 16 anos aumenta em 1,71 vezes a chance de ter melhor QV no domínio relacionado à autonomia e relação com os pais. Depois disso, verifica-se o aumento das preocupações, da percepção da responsabilidade da escolha de uma profissão e da necessidade de independência financeira dos pais com o aumento progressivo da idade, o que pode interferir na QV e na felicidade ${ }^{(32)}$.

No presente estudo, adolescentes de classe econômica D têm 1,62 vezes mais chances de ter melhor QV no domínio relacionado aos amigos e apoio social do que os da classe econômica E. Estudantes com condições socioeconômicas mais elevadas apresentam indicadores sociais e de saúde pessoal mais positivos, são mais otimistas, apresentam melhor autoestima e também maior satisfação com o suporte social, relacionado com os pais e os amigos ${ }^{(33)}$. Assim, o apoio social também é considerado como um fator de proteção para a saúde de crianças e adolescentes ${ }^{(34)}$.

Em um estudo para avaliar a QV de adolescentes de escolas do município de São Paulo, com uma amostra de 2.434 estudantes, observou-se que vários aspectos influenciaram na QV. O sexo masculino, de menor idade, com renda maior ${ }^{(35,36)}$ e a prática de atividade física impactaram positivamente na QV dessa população.

Pôde-se obervar interferência do curso na QV dos adolescentes investigados na presente pesquisa, uma vez que os adolescentes que cursam Eletrotécnica têm 3,27, 2,23 e 2,27 vezes mais chances, respectivamente, de ter melhor QV no domínio autonomia e relação com os pais, no domínio amigos e apoio social e melhor QV total do que os de Química. A maioria dos adolescentes pertencem às classes $D$ e $E$, nas quais a independência financeira é algo extremamente almejado por eles. Assim, a oferta de um curso aos alunos de Eletrotécnica aumenta a chance de ingresso precoce no mercado de trabalho, que necessita de pessoas capacitadas para responder ativamente às demandas exigidas ${ }^{(37)}$.

A felicidade subjetiva demonstrou-se uma variável importante a ser considerada no atual estudo, pois a melhor percepção de felicidade aumentou em 7,10 vezes a chance de ter maior QV. Esse resultado observado pode ser explicado pelo fato de a maioria dos adolescentes elencar a felicidade como algo almejado para lidar com as demandas da vida de forma mais positiva e adaptativa, destacando a escola, a família e a comunidade como principais ambientes relacionados a essa felicidade ${ }^{(38,39)}$.

Observou-se, também, uma grande interferência da felicidade no domínio relacionado ao bem-estar psicológico, aumentando em 15,74 vezes a chance de ter maior QV. O bem-estar psicológico é um conceito multifuncional que requer o desenvolvimento positivo das capacidades que contribuem para o pleno funcionamento das potencialidades de um individuo, envolvendo a sua capacidade de pensar e de bom-senso(40).

Considerou-se um fator limitante do presente estudo a natureza transversal da avaliação da percepção, mostrando os resultados de forma momentânea e pontual. Outro aspecto refere-se ao possível viés de seleção, tendo em conta o fato de ter-se uma amostra de apenas uma escola, o que pode não refletir a realidade da população adolescente da região. Mas, em contraponto, ajuda a compreender a realidade de um grupo exposto ao ensino diferenciado e de qualidade oferecido pelo IF Sertão-PE. Pode-se citar, ainda, a desejabilidade social, já que nessa fase da vida os adolescentes podem ter optado por respostas consideradas mais socialmente desejadas, entretanto a aplicação de um questionário criado de forma específica para a população adolescente, com rigor metodológico, usado em vários países e com níveis elevados de validade e fidedignidade diminui esse fenômeno.

A adolescência, por se tratar de um período complexo da vida, necessita de politicas públicas que abordem os vários aspectos necessários para promoção de saúde nesse grupo específico, e a escola, no seu papel de instituição de acolhimento, escuta. Mas o reconhecimento das mudanças e das situações de risco é extrememente importante na constituição de uma rede de cuidados singulares para que efetivamente se possa promover uma melhor QV nessa população(34).

Todos os achados acima discutidos evidenciam a necessidade de sempre vilumbrar o desenvolvimento de ações intersetoriais, as quais devem envolver a escola, a comunidade e a família, que são fundamentais no desenvovimento de ambientes saudáveis para melhorar o bem-estar e a QV dessa população(6).

\section{CONCLUSÃO}

Ser do sexo masculino, estar matriculado no curso de Eletrotécnica e relatar felicidade subjetiva influenciaram positivamente a qualidade de vida. Sendo a escola o espaço social mais presente na vida dos adolescentes, tornase um local privilegiado para desenvolvimento de ações intersetoriais relacionadas ao cuidado com a vida e à promoção de saúde de forma a minimizar suas vulnerabilidades. 


\section{CONFLITOS DE INTERESSE}

Os autores informam que não há conflitos de interesses em decorrência da realização deste estudo.

\section{CONTRIBUIÇÕES}

Flávia Martão Flório e Luciane Zanin de Souza contribuíram com a elaboração e delineamento do estudo; a aquisição, análise e interpretação dos dados e a redação e/ou revisão do manuscrito. Adália Maria Dias Palma Leal contribuiu com a elaboração e delineamento do estudo e a redação e/ou revisão do manuscrito.

\section{REFERÊNCIAS}

1. Santos NC, Abdala GA. Religiosidade e qualidade de vida relacionada à saúde dos idosos em um município na Bahia, Brasil. Rev Bras Geriatr Gerontol. 2014;17(4):795-5.

2. Pardo-Guijarro MJ, Martínez-Andrés M, Notario-Pacheco B, Solera-Martínez M, Sánchez-López M, MartínezVizcaíno V. Self-reports versus parental perceptions of health-related quality of life among deaf children and adolescents. J Deaf Stud Deaf Educ. 2015;20(3):275-82.

3. Sawyer SM, Azzopardi PS, Wickremarathne D, Patton GC. The age of adolescence. Lancet Child Adolesc Health. 2018;2(3):223-8.

4. Presidência da República (BR). Lei n 8.069, de 13 de julho de 1990. Dispõe sobre o Estatuto da Criança e do Adolescente, e dá outras providências. Brasília: Presidência da República; 1990.

5. Fundação Abrinq. Cenário da Infância e Adolescência no Brasil. São Paulo: Fundação Abrinq; 2019.

6. Campos HM, Schall VT, Nogueira MJ. Saúde sexual e reprodutiva de adolescentes: interlocuções com a Pesquisa Nacional de Saúde do Escolar (PeNSE). Saúde Debate. 2013;37(97):336-346.

7. Ministério da Saúde (BR), Secretaria de Atenção em Saúde, Departamento de Ações Programáticas Estratégicas. Área técnica de saúde do adolescente e do jovem. Diretrizes nacionais para a atenção integral à saúde de adolescentes e jovens na promoção, proteção e recuperação da saúde. Brasília: Ministério da Saúde; 2010.

8. Ministério da Saúde (BR), Secretaria de Atenção em Saúde, Departamento de Ações Programáticas Estratégicas. Diretrizes nacionais para a atenção integral à saúde de adolescentes e jovens na promoção, proteção e recuperação da saúde. Brasília: Ministério da Saúde; 2010.

9. Malta DC, Morais OL Neto, Silva MMAD, Rocha D, Castro AMD, Reis AACD, et al. Política Nacional de Promoção da Saúde (PNPS): capítulos de uma caminhada ainda em construção. Ciênc Saúde Colet. 2016;21:1683-94.

10. Zamboni GLP, Lima RL, Duarte DA, Sant'Anna GR. Percepções, conhecimentos e representações de saúde bucal em adolescentes de escolas públicas e privadas do município de Atibaia, SP. RFO UPF. 2015;20(2):179-86.

11. Vasconcelos ACM, Oliveira KMC, Rocha NNV, Cavalcante JHV. O protagonismo dos adolescentes na escola: tecendo a rede psicossocial álcool, crack e outras drogas. Sanare Sobral. 2015;14(2):117-22.

12. Alves MAR, Pinto GMC, Pinto MHB, Pedroso B. Um levantamento quantitativo da utilização do instrumento Kidscreen na avaliação da qualidade de vida de crianças: uma revisão na produção científica utilizando a base de dados Scopus. Rev Interdisciplin Estudos Saúde. 2019;8(2):15-25.

13. Portella MR, Scortegagna EIM, Pichler NA, Graeff DB. Felicidade e satisfação com a vida: voz de mulheres adultas e idosas. Rev Bras Ciênc Envelhec Hum. 2017;14(1):93-101.

14. Pereira D, Araújo UF. Uma reflexão sobre a busca e o significado da felicidade. Rev Educ Linguagens. 2018;7(12):17-31.

15. Instituto Federal de Educação, Ciência e Tecnologia do Sertão Pernambucano. Relatório de Gestão do Exercício de 2018. Recife: IF Sertão Pernambucano; 2019. 
16. Lyubomirsky S, Heidi SL. A measure of subjective happiness: Preliminary reliability and construct validation. Soc Indic Res. 1999;46(2):137-55.

17. Ravens-Sieberer U, Herdman M, Devine J, Otto C, Bullinger M, Rose M, et al. The European KIDSCREEN approach to measure quality of life and well-being in children: development, current application, and future advances. Qual Life Res. 2014;23(3):791-803.

18. Sobral ME, Gontijo DT, Abdala DW, Cabral TN. Avaliação da qualidade de vida de adolescentes em situação de vulnerabilidade social. Rev Bras Promoc Saúde. 2015;28(4):568-77.

19. Assis TL, Martins JS, Silva FGC. Percepção da qualidade de vida em escolares do ensino médio da cidade de Fortaleza, Ceará. Ciênc Mov. 2017;19(38):39-46.

20. Brito US, Rocha EMB. Percepção de jovens e adolescentes sobre saúde e qualidade de vida. Rev Bras Promoç Saúde. 2019;32:8933.

21. Molina T, Montaño RE, González EA, Sepúlveda RP, Hidalgo-Rasmussen C, Martínez VN, et al. Propiedades psicométricas del cuestionario de calidad de vida relacionada con la salud KIDSCREEN-27 en adolescentes chilenos. Rev Méd Chile. 2014;142(11): 1415-21.

22. Quiceno JM, Vinaccia S. Calidad de vida, fortalezas personales, depresión y estrés en adolescentes según sexo y estrato. Int J Psychol Psychol Ther. 2014;14(2):155-70.

23. Agathão $B T$, Reichenheim ME, Moraes CL. Qualidade de vida relacionada à saúde de adolescentes escolares. Ciênc Saúde Colet. 2018;23(2):659-68.

24. Maria AT, Guimarães C, Candeias I, Almeida S, Figueiredo C, Pinheiro A, et al. Qualidade de Vida Relacionada com a Saúde em Adolescentes Portugueses: Estudo Numa População Escolar. Acta Pediatr Port. 2017;48(3):203-11.

25. Rubin KH, Bukowski W M, Bowker JC. Children in peer groups. In: Bornstein MH, Leventhal T, Lerner RM. Handbook of child psychology and developmental science: Ecological settings and processes Hoboken. New Jersey: John Wiley; 2015. p. 175-222.

26. Tomé G, Gómez-Baya D, Cerqueira A, Matos MG. Que escola é precisa para aprender, sem pôr em perigo o bem-estar e as relações interpessoais dos adolescentes. Rev Pensamento Conteporâneo Adm. 2019;10(1):63-73.

27. Higuita-Gutiérrez LF, Cardona-Arias JA. Evaluation instruments to assess quality of life related to adolescent's health's. Hacia Promoc Salud. 2015;20(2):27-42.

28. Gomez-Baya D, Mendoza R, Paino S, Gillham JE. A two-year longitudinal study of gender differences in responses to positive affect and depressive symptoms during middle adolescence. J Adolesc. 2017;56:11-23.

29. Wu H, Wu S, Wu H, Xia Q, Li N. Living arrangements and health-related quality of life in Chinese adolescents who migrate from rural to urban schools: mediating effect of social support. Int J Environ Res Public Health. 2017;14(10):1-10.

30. Cureau FV, Silva TLN, Bloch KV, Fujimori E, Belfort DR, Carvalho KMB, et al. ERICA: inatividade física no lazer em adolescentes brasileiros. Rev Saúde Pública. 2016;50(Supl. 1):1-11.

31. Bloch KV, Kuschnir MC, Szklo M. Cardiovascular risk in adolescence. Cad Saúde Pública. 2012;28(7):1220-1.

32. Bolton K, Kremer P, Rossthorn N, Moodie M, Gibbs L, Waters E, et al. The effect of gender and age on the association between weight status and health-related quality of life in Australian adolescents. BMC Public Health. 2014;14:898.

33. Gaspar T, Balancho L. Fatores pessoais e sociais que influenciam o bem-estar subjetivo: diferenças ligadas estatuto socioeconômico. Ciênc Saúde Colet. 2017;22(4):1373-80.

34. Gomes AC. Condição socioeconômica, apoio social, fatores psicossociais, comportamentos em saúde e qualidade de vida relacionada à saúde em adolescentes: um estudo de seguimento de 6 meses [dissertação]. Manaus: Universidade Federal do Amazonas; 2019.

35. Benincasa M, Custódio EM. Avaliação da qualidade de vida em adolescentes do município de São Paulo. Bol Psicol. 2011;61(134):31-42. 
36. Silva BVS, Andrade PMC, Baad VMA, Valença PAM, Menezes VA, Amorim VC, et al. Prevalence of and factors associated with the adolescents' negative self-perception in health: a systematic review. Rev Bras Promoç Saúde. 2016:29(4):595-601.

37. Ferreira CCJ, Menezes L, Barbosa MV, Lucas GAP. Influência do curso técnico pela escolha da graduação em administração. Rev Valore. 2017;2(1):8-33.

38. Lima RFF, Morais NA. Bem-estar subjetivo de crianças e adolescentes: revisão integrativa. Ciênc Psicol. 2018;12(2): 249-60.

39. Pinto AVDL, Cavalcanti JG, Araújo LSD, Coutinho MDL, Coutinho MDPDL. Depressão e adolescência: relação com qualidade de vida e bem-estar subjetivo. Rev Psicol IMED. 2018;10(2):6-21.

40. Silva DG, Giordani JP, Dell'Aglio DD. Relações entre satisfação com a vida, com a família e com as amizades e religiosidade na adolescência. Est Inter Psicol. 2017;8(1):38-54.

Endereço do primeiro autor:

Adália Maria Dias Palma Leal

Faculdade São Leopoldo Mandic

Rua José Rocha Junqueira, 13

Bairro: Ponte Preta

CEP: 13041-445 - Campinas - SP - Brasil

E-mail: adaliapalma@yahoo.com.br

\section{Endereço para correspondência:}

Luciane Zanin de Souza

Faculdade São Leopoldo Mandic

Rua José Rocha Junqueira, 13

Bairro: Ponte Preta

CEP: 13041-445 - Campinas - SP - Brasil

E-mail: zaninsouza@yahoo.com.br

Como citar: Leal AMDP, Flório FM, Souza LZ. Relação entre qualidade de vida e felicidade subjetiva de adolescentes escolares. Rev Bras Promoç Saúde. 2020;33:10159. 